\title{
Ecm1 is a new pre-ribosomal factor involved in pre-60S particle export
}

\author{
YANHUA YAO, ${ }^{1}$ EMILIE DEMOINET, ${ }^{1,2}$ COSMIN SAVEANU, ${ }^{1}$ PASCAL LENORMAND, ${ }^{3}$ ALAIN JACQUIER, ${ }^{1}$ \\ and MICHELINE FROMONT-RACINE ${ }^{1}$ \\ ${ }^{1}$ Institut Pasteur, Unité de Génétique des Interactions Macromoléculaires, CNRS-URA2171, F-75724 Paris Cedex 15, France \\ ${ }^{2}$ Défenses Antivirales et Antitumorales, UMR5235 cc107, Université Montpellier 2, 34095 Montpellier cedex 5, France \\ ${ }^{3}$ Institut Pasteur, Plate-forme Protéomique, F-75724 Paris cedex 15, France
}

\begin{abstract}
In eukaryotes, ribosome biogenesis is a highly conserved process that starts in the nucleus and ends in the cytoplasm. In actively growing yeast cells, it is estimated that each nuclear pore complex (NPC) contributes to the export of about 25 pre-ribosomal particles per minute. Such an extremely active process requires several redundant export receptors for the pre-60S particles. Here, we report the identification of a novel pre-60S factor, Ecm1, which partially acts like Arx1 and becomes essential when the NPC function is affected. Ecm 1 depletion, combined with the deletion of NPC components led to pre-60S retention in the nucleus. Functional links that we identified between Ecm1, 60S biogenesis, pre-60S export, and the NPC were correlated with physical interactions of Ecm 1 with pre-60S particles and nucleoporins. These results support that Ecm 1 is an additional factor involved in pre-60S export. While Ecm1 and Arx1 have redundant functions, overproduction of either one could not complement the absence of the other, whereas overproduction of Mex67 was able to partially restore the growth defect resulting from the absence of Ecm1 or Arx1. These data highlight the involvement of many factors acting together to export pre605 particles.
\end{abstract}

Keywords: ribosome biogenesis; pre-60S export; karyopherins; nucleoporin; yeast Saccharomyces cerevisiae

\section{INTRODUCTION}

In eukaryotic cells, ribosome biogenesis starts within the nucleolus with the transcription of the $35 \mathrm{~S}$ rRNA and the pre-5S rRNA precursors by RNA Pol I and RNA Pol III, respectively. The association of rRNA precursors with ribosomal and pre-ribosomal proteins and with U3 snoRNP forms a first intermediate, the $90 \mathrm{~S}$ or SSU-processome (Dragon et al. 2002; Grandi et al. 2002). Several successive endo- and exo-nucleolytic cleavages of the 35S rRNA precursor take place within these pre-ribosomal particles and lead to various intermediate rRNAs (Venema and Tollervey 1999; Fromont-Racine et al. 2003). A major rRNA cleavage gives rise to the pre-60S and pre-40S particles formed by specific pre-ribosomal factors. Following nuclear maturation, the pre-60S and pre-40S pre-ribosomal subunits are independently exported to the cytoplasm through the nu-

Reprint requests to: Micheline Fromont-Racine, Institut Pasteur, Unité de Génétique des Interactions Macromoléculaires, CNRS-URA2171, 25 rue du docteur Roux, 75724 Paris Cedex 15, France; e-mail: mfromont@ pasteur.fr; fax: 33145688790.

Article published online ahead of print. Article and publication date are at http://www.rnajournal.org/cgi/doi/10.1261/rna.2012310. clear pore complex (NPC), where they undergo final maturation to give rise to the large and small ribosomal subunit, respectively (Johnson et al. 2002; Tschochner and Hurt 2003; Zemp and Kutay 2007; Henras et al. 2008).

NPCs are the gates that mediate trafficking of large molecules and complexes through the nuclear envelope. In Saccharomyces cerevisiae, the NPC is composed of at least 30 different nucleoporins (Nups) present in many copies that form an octagonal structure of $\sim 50 \mathrm{MDa}$. The NPC's core scaffold is formed by the outer (Nup84 complex) and inner (Nup188, Nup192, Nup170, Nup157) rings. The NPC is anchored to the pore membrane via the membrane ring formed by three proteins (Pom34, Pom152, and Ndc1). Nucleocytoplasmic trafficking is highly dynamic and is mediated by FG nucleoporins (Tran and Wente 2006). These proteins expose FG repeat regions, which are the docking sites for the transport of cargo factors. Some of these FG nucleoporins are attached to the cytoplasmic (Nup42, Nup100, Nup116, and Nup159) or nucleoplasmic (Nup60, Nup145N, and Nup1) side of the NPC, while others are found symmetrically on both sides (Nup49, Nup53, Nup57, Nup59, and Nsp1) (Rout et al. 2000; Fahrenkrog and Aebi 2003; Tran and Wente 2006; Alber et al. 2007a,b). 
Since about 2000 ribosomes are synthesized every minute in growing yeast cells, a very efficient transport process is required to export about 25 pre-ribosomal particles through each NPC per minute (Warner 1999). Simultaneously, a very efficient ribosomal and pre-ribosomal protein import is necessary. About 1000 ribosomal proteins must be imported per NPC and per minute (Warner 1999). This high level of nucleocytoplasmic trafficking involves specific cargo carriers (karyopherins), which interact with the FG nucleoporins. This interaction with the cargo is regulated by the complex formed with the GTP-bound state of the Ran-GTPase. There are 10 known members of the yeast karyopherin family that function as importins. A redundancy of function was observed between these carriers. For example, the karyopherins $\beta 3$ (Kap121) and $\beta 4$ (Kap123) are both involved in the import of ribosomal proteins and histones $(\mathrm{H} 2 \mathrm{~A} / \mathrm{H} 2 \mathrm{~B})$ (Sydorskyy et al. 2003).

Redundancy is also observed for many export processes. Recent studies have shown that the export of 605 subunits is mediated by several export receptors. The first identified nuclear export receptor was Crm1. It recognizes an adapter, Nmd3, which binds the pre-60S and contains leucine-rich nuclear export signal (NES). Notably, it has been shown that a crm 1 mutant in which the interaction with the NES is disrupted blocks pre-60S export (Ho et al. 2000; Gadal et al. 2001). The mRNA export receptor Mex67-Mtr2 has been further shown to play a role in the large subunit export (Yao et al. 2007). This heterodimer contains a loop confined surface in the NTF2-like scaffold that seems to play a central role for the functions of the complex both in pre-60S and mRNA export (Yao et al. 2008). More recently, a third export receptor, the shuttling factor Arx1, was identified, demonstrating a high level of redundancy for the pre-60S export machinery (Bradatsch et al. 2007; Hung et al. 2008). These multiple pre-ribosomal nuclear export receptors might cooperate to ensure an efficient pre-60S export to the cytoplasm.

To determine if other factors are involved in pre-60S nucleocytoplasmic transport, we first performed genetic screens with an arx $1 \Delta$ strain and next secondary screens with gene deletions that exhibited a synthetic growth defect in combination with the absence of $A R X 1$. We thus identified ecm $1 \Delta$, which exhibits a range of genetic interactions very similar to $\operatorname{ar} x 1 \Delta$ when compared to 39 other large-scale genetic screens performed with mutants involved in RNA metabolism (Decourty et al. 2008). Ecm1 was previously identified as synthetic lethal with nug1-1 and mtr2-33 alleles (Bassler et al. 2001). We show here that Ecm1 associates with pre-60S particles and binds to nucleoporins. The gene becomes essential when the nuclear pore is affected, leading to nuclear retention of the nascent $60 \mathrm{~S}$ subunits under such conditions. These results indicate that Ecm 1 is an additional factor that facilitates the nuclear export of pre-60S particles.

\section{RESULTS}

\section{Ecm1 and Arx1 are functionally linked to nucleocytoplasmic transport}

Arx1 was recently described as a novel pre-60S particle export receptor (Bradatsch et al. 2007; Hung et al. 2008). To identify additional factors involved in this process, we looked for functional partners of Arx1 by analyzing the results of 41 genome-wide genetic screens using the GIM method. This method allows the identification of specific genetic interaction by measuring competitive growth in liquid medium of a pool of double mutants (Decourty et al. 2008). Among these 41 large-scale genetic screens, most of the different query mutations have been chosen within genes involved in several RNA metabolic pathways. Inspection of these data revealed a similar genetic interaction profile for arx $1 \Delta$ and ecm $1 \Delta$. These two deletion mutant strains have an aggravating phenotype in combination with several deletions for the same subset of genes (Fig. 1A). Interestingly, the strongest synthetic lethal candidates of both arxis and ecm $1 \Delta$ were the FG nucleoporins (NUP2, NUP42, NUP59) and components of the NPC membrane ring mutants (POM34 and POM152) (Fig. 1A). These large-scale screen results are thus in excellent agreement with the results that linked Arx1 to FG nucleoporins (Bradatsch et al. 2007; Hung et al. 2008). Looking carefully to the other synthetic lethal candidates, we observed that three of these deletions were directly adjacent to essential genes involved in the nucleocytoplasmic pathway (MEX67 and NTF2) or in pre-ribosome biogenesis (NAF1) (Fig. 1A). The observed phenotype might result from a perturbation of the neighboring essential gene rather than by the lack of the deleted ORF itself as previously observed (Decourty et al. 2008) and see below for MEX67. Interestingly, an ecm 1 mutant strain was previously identified as being synthetic lethal with $m t r 2-33$, a nucleoplasmic transport factor mutant partner of Mex67 (Bassler et al. 2001). Moreover, ECM1 deletion was recently shown to be synthetic lethal with the deletion of $A R X 1$ (Bradatsch et al. 2007).

To validate these results, serial dilutions were performed to individually test synthetic lethal or synthetic slow growth candidates related to the nucleocytoplasmic pathway. Because some of these double deletion combinations are lethal, we constructed strains in which ECM1, under the control of the GAL1 promoter $\left(P_{G A L 1}-E C M 1\right)$, is associated to the deletion of each gene candidate. The growth of each double-mutant strain was tested in repressive rich glucose medium (YPD). A strong synthetic growth defect was observed when combining $P_{G A L 1}$-ECM1 with pom34s, nup2s, or $\operatorname{arx} 1 \Delta$ (Fig. 1B,C).

Altogether, the genome-wide genetic screens and the individual validations revealed that ECM1 is embedded in a network of functional interactions focused on the nuclear pore. It exhibits a genetic interaction profile very similar to $A R X 1$, and its deletion is synthetic lethal with $\operatorname{arx} 1 \Delta$. 


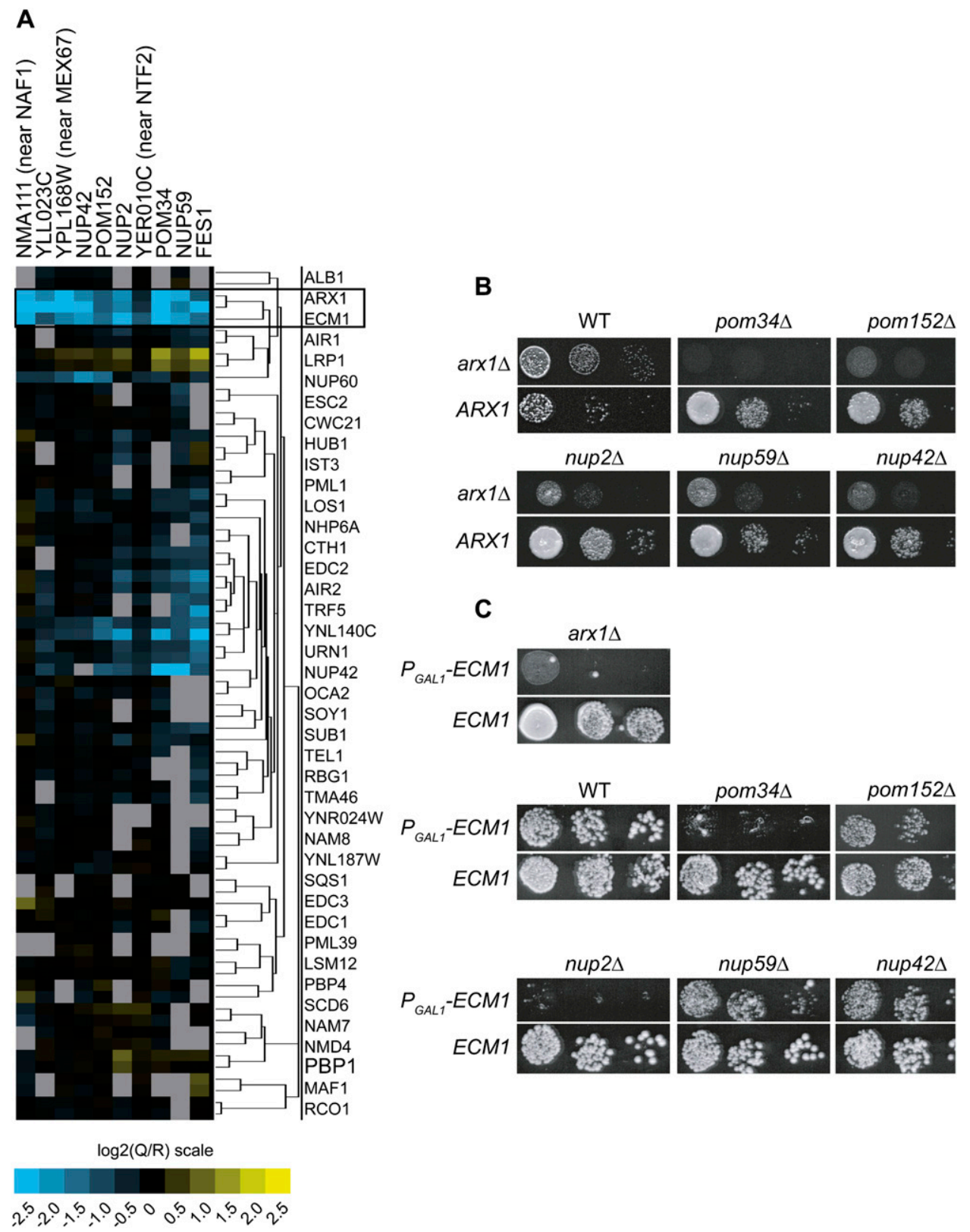

FIGURE 1. ECM1 and ARX1 exhibit genetic interactions. (A) Two-dimensional hierarchical cluster plot of double-mutant profiles from the GIM genetic screens. Query mutations are represented on the horizontal axis and the selected candidates on the vertical axis (in blue for the aggravating phenotype). Two screens were performed with arxls and one screen with ecm $1 \Delta$ strains as query mutants. Normalized results are expressed as $\log _{2}(\mathrm{Q} / \mathrm{R})$ ( $\mathrm{Q}$ for the query mutant strain and R for the reference strain) (Decourty et al. 2008). (B) Serial dilutions of the double mutants obtained by

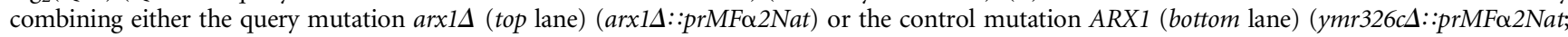
taken here as a wild-type reference) (Decourty et al. 2008) with some of the deletion mutants, identified in the GIM genetic screen. After mating and diploid selection followed by sporulation, the cells were spotted on YPD (glucose medium) plates containing geneticin and nourseothricin to select the haploid double mutants and incubated for $2 \mathrm{~d}$ at $30^{\circ} \mathrm{C}$. $(C)$ Serial dilutions of double mutants obtained by combining either the query mutation

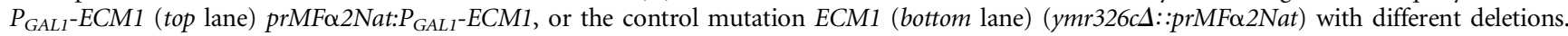
After mating and diploid selection followed by sporulation, double mutant strains were selected on YPGal (galactose medium) containing geneticin and nourseothricin and isolated. To repress ECM1 expression, the cells were spotted on YPGlu plates and incubated for $3 \mathrm{~d}$ at $30^{\circ} \mathrm{C}$.

\section{Ecm1 becomes essential for ribosome biogenesis when the nuclear pore is affected}

Since ECM1 was functionally linked to nucleoporins and to the pre-60S export receptor Arxl, we addressed the question of Ecml involvement in the nucleocytoplasmic transport of the pre-60S subunit. We analyzed the nuclear export of the large ribosomal subunit using Rpl25-eGFP as a reporter in different mutants (Fig. 2A). The ecml $1 \Delta$ mutant strain, as well as the pom $34 \Delta$ or $\operatorname{arx} 1 \Delta$ mutant 


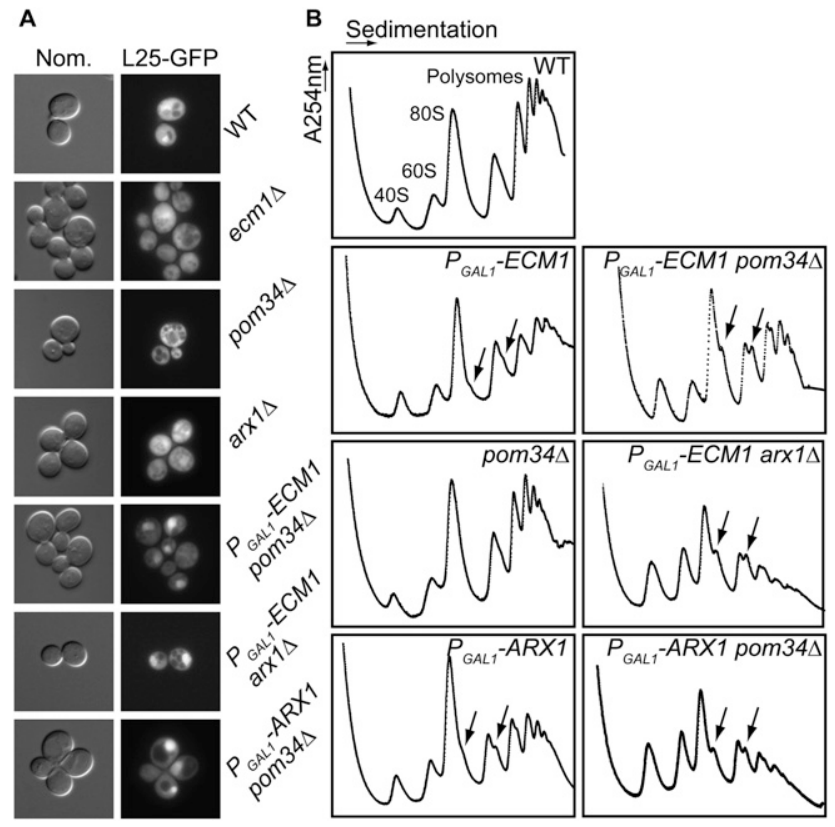

FIGURE 2. Ecm1 becomes essential for ribosome biogenesis when the nuclear pore is affected. (A) In the absence of Pom34 or Arx1, depletion of Ecml leads to pre-60S accumulation in the nucleus. Subcellular localization of Rpl25-eGFP (60S reporter) was observed by fluorescence microscopy. The double mutants $\left(P_{G A L 1}-E C M 1\right.$ arx1S,

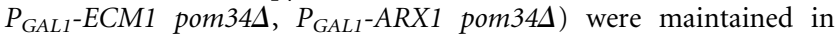
galactose-rich medium (YPGal) and shifted to glucose containing rich medium (YPD) for $17 \mathrm{~h}$ at $30^{\circ} \mathrm{C}$ (time at which the growth rate begins to decrease). (B) The absence of Ecml leads to $60 \mathrm{~S}$ biogenesis defects when the nuclear pore is affected. All the strains were incubated for $17 \mathrm{~h}$ in YPD liquid medium. The mutants, having one gene under the control of the GAL1 promoter, were maintained in YPGal medium prior to the $17 \mathrm{~h}$ of incubation in YPD. Whole cellular

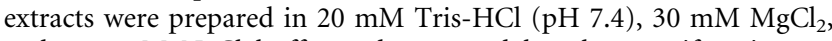
and $100 \mathrm{mM} \mathrm{NaCl}$ buffer and separated by ultracentrifugation on a sucrose gradient $(10 \%-50 \%)$. The position of the $40 \mathrm{~S}$ subunit, $60 \mathrm{~S}$ subunit, $80 \mathrm{~S}$ ribosomes, and polysomes are noted, and arrows indicate halfmers.

strains, exhibited a cytoplasmic localization of Rpl25-eGFP that was not different from a wild-type (WT) strain. In contrast, $P_{G A L 1}-E C M 1$ pom34A, $P_{G A L 1}-E C M 1$ arx $1 \Delta$, and $P_{G A L 1}-A R X 1$ pom34s double mutants accumulated Rpl25eGFP in the nucleus under non-permissive growth conditions (17 h of growth in glucose medium, after a preculture in galactose medium). These results seem specific to the $60 \mathrm{~S}$ export since we did not observe any nuclear accumulation of pre-40S particles when using an Rps2-GFP reporter gene as a control (data not shown). These results strongly support the hypothesis that Ecml is involved in pre-60S export and plays a role similar to and redundant with Arx1.

We asked therefore if the observed pre-60S export defects were associated with a defect in $60 \mathrm{~S}$ biogenesis in the absence of Ecm1. We analyzed polysome profiles by sucrose gradient ultracentrifugation under non-permissive conditions for the three double mutants $P_{G A L 1}-E C M 1$ pom34A, $P_{G A L 1}-E C M 1$ arx $1 \Delta$, and $P_{G A L 1}-A R X 1$ pom34s in comparison to the corresponding single mutants (Fig. 2B). The depletion of Ecm1, like its absence (data not shown), led to weak but reproducible formation of halfmers, reflecting a relative deficit in $60 \mathrm{~S}$ as compared to $40 \mathrm{~S}$ in the cytoplasm during translation initiation, whereas in the absence of Pom34, for example, the polysome profile was similar to that of a WT strain (Fig. 2B). As expected, the depletion of Arxl did not significantly affect polysome profile but led to the appearance of halfmers, consistent with previous results obtained in the absence of Arxl (Lebreton et al. 2006; Demoinet et al. 2007). In contrast, in the three double mutants $P_{G A L 1}-E C M 1$ pom $34 \Delta, P_{G A L 1^{-}}$ ECM1 arx $1 \Delta$, and $P_{G A L 1}-A R X 1$ pom34D, we observed a decrease of the $60 \mathrm{~S}$ large subunit levels, a decrease of polysomes, and the presence of halfmers. We measured the ratio between $40 \mathrm{~S}$ and $60 \mathrm{~S}$ particles in the different mutant strains in comparison with a wild-type strain by performing sucrose gradient analyses under conditions that disrupt the polysomes and the $80 \mathrm{~S}$ particles. Whereas the $60 \mathrm{~S} / 40 \mathrm{~S}$ ratio was around 2 in the WT strain, the $60 \mathrm{~S}$ subunit amount was lower in all the double mutants under nonpermissive conditions $\left(1.48 \pm 0.2\right.$ in $P_{G A L 1}-E C M 1, \operatorname{arx} 1 \Delta, 1.63 \pm 0.14$ in $P_{G A L 1}-A R X 1, p o m 34 \Delta$, and of $1.7 \pm 0.18$ for $P_{G A L 1^{-}}$ ECM1, pom 34s).

In parallel with the decrease of the $60 \mathrm{~S} / 40 \mathrm{~S}$ ratio, a slight decrease of the $25 \mathrm{~S}$ mature rRNA and a weak accumulation of the $27 \mathrm{SB}$ intermediate were observed in the doublemutant strains in comparison to either the wild-type or the $\operatorname{arx} 1 \Delta$, ecm $1 \Delta$, or pom $34 \Delta$ single-mutant strains (data not shown). This slight change in 27SB pre-rRNA levels is frequently observed in most of the strains defective in large ribosomal subunit assembly or export and is probably due to an indirect feedback effect (Venema and Tollervey 1999; Fromont-Racine et al. 2003).

In conclusion, even if the absence of Ecm 1 does not give rise to a cell growth defect, the role of Ecm 1 becomes essential for the export of the pre-60S subunit when the nuclear pore is affected or when the pre-60S export receptor, Arx1, is absent.

\section{Ecm1 is associated with the pre-60S particles}

Altogether, our results suggest that Ecm1 could be a pre$60 \mathrm{~S}$ factor involved in the export of pre-ribosomal particles, but the only reported physical associations of Ecm1 involve karyopherins, such as Kap123, Kap108/Sxm1, and Kap121/Pse1 (Gavin et al. 2006). To determine whether Ecm 1 might be present or might interact with pre-ribosomal particles, we purified the complex associated to a TAP-tagged version of Ecml according to Rigaut et al. (1999). For comparison, we purified the complex associated with Arx1 (strain Arx1-TAP, Ecm1-3HA). A strain only expressing Ecm1-3HA fusion protein was used as negative control. We further tested the functionality of both Ecm1-TAP and Ecm1-3HA fusion proteins. Since the 
deletion of ECM1 is viable but became essential in the absence of Arxl, we tested the viability of either Ecm1-TAP or Ecm1-3HA in combination with arx1s (Supplemental Fig. 1A). We compared the growth of these double-mutant strains with WT and simple mutants. We also checked polysome profiles (Supplemental Fig. 1B). The Ecm1-TAP fusion protein in absence of Arx1 leads to a weak growth defect, but no significant effect was observed on the polysome profile in comparison with an $\operatorname{arx} 1 \Delta$ strain, whereas Ecm1-3HA fusion protein has no defect phenotype.

Components of Ecm1-TAP and Arx1-TAP purified complexes were separated on denaturing polyacrylamideSDS gels and stained with colloidal Coomassie Blue (Fig. 3A). Both Ecm1- and Arx1-associated complexes present similar electrophoteric profiles, characteristic of the pre-60S particles. The protein composition of the Ecm1-associated com-
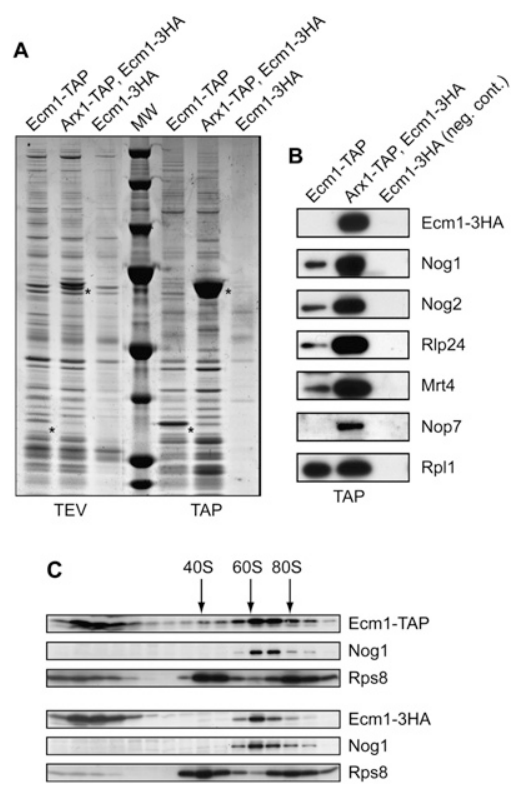

FIGURE 3. Ecml is associated with pre-60S complexes. (A) Ecm1TAP complex purification. Proteins associated with Ecm1-TAP (strain Ecm1-TAP) and Arx1-TAP (strain Arx1-TAP, Ecm1-3HA) were separated on a $4 \%-12 \%$ SDS-polyacrylamide gel and stained with colloidal Coomassie Blue. The strain used as negative control expressed Ecm1-3HA only. Asterisks indicate the bait proteins fused to CBP. Proteins associated with Ecml-TAP complexes were identified by mass spectrometry (see Table 1). (B) Ecml co-purifies specifically with pre-60S factors. Associated proteins with the $\mathrm{Ecm} 1$ and Arxl complexes after TAP purification were separated on a denaturating $10 \%$ polyacrylamide-SDS gel. The presence of preribosomal factors and ribosomal proteins was determined by immunoblotting with specific antibodies. $(C) \mathrm{Ecm} 1 \mathrm{co-sediments} \mathrm{with} \mathrm{the}$ $60 \mathrm{~S}$ peak. Cellular extracts from strains expressing either Ecm1-TAP or Ecm1-3HA fusion proteins were prepared in buffer containing 20 $\mathrm{mM}$ Tris (pH 7.4), $50 \mathrm{mM} \mathrm{KCl}$, and $5 \mathrm{mM} \mathrm{MgCl}_{2}$, and separated on a sucrose gradient $(10 \%-50 \%)$. Sixteen fractions were collected, and proteins were TCA-precipitated. After separation on a denaturating $10 \%$ polyacrylamide-SDS gel, Ecm1-TAP, Ecm1-3HA, Nog1, and Rps8 were revealed by Western blot using PAP or HA antibodies and Nog1 and Rps8 antibodies, respectively. Peaks corresponding to the $40 \mathrm{~S}, 60 \mathrm{~S}$, and $80 \mathrm{~S}$ particles are indicated. plex was determined by mass spectrometry. Three pre-60S factors-Nog1, Nog2, Fpr4-and the karyopherins Kap121 and Kap108 were identified (Table 1). We and others previously found Fpr4 biochemically associated with several different pre-60S factors by TAP-tagging experiments (Saveanu et al. 2003; Gavin et al. 2006). Ypr108w-a (7.7 $\mathrm{kDa})$, a protein of unknown function, was also identified. In addition to in silico evidence and RT-PCR data (Kessler et al. 2003), this is the first evidence for the existence of this protein. Large and small ribosomal proteins and several abundant translation factors were also identified but are common contaminants of TAP purifications. We confirmed the association of Ecm 1 with factors of pre-60S particles by Western blot analysis using a number of antibodies against pre-ribosomal and ribosomal proteins (Fig. 3B). As expected, Nog1 and Nog2 were present in both complexes. Other late pre-60S factors such as Mrt4 and Rlp24 were also detected, while Nop7 was absent from the Ecm1-TAP complex. These results revealed that mass-spectrometry analysis was not exhaustive. To confirm that Ecm 1 was part of pre$60 \mathrm{~S}$ particles, we performed sucrose gradients with cellular extracts from the strain expressing either Ecm1-TAP or Ecm1-3HA fusion proteins. Using buffer containing $20 \mathrm{mM}$ Tris (pH 7.4), $50 \mathrm{mM} \mathrm{KCl}$, and $5 \mathrm{mM} \mathrm{MgCl}_{2}, \mathrm{Ecm} 1$-TAP and Ecm1-3HA mainly co-sedimented with Nog1 in the 60S peak (Fig. 3C). However, using buffer containing $30 \mathrm{mM}$ $\mathrm{MgCl}_{2}, \mathrm{Ecm} 1$ fusion proteins sedimented in the first fractions at the top of the gradient (data not shown). A comparative study using either $5 \mathrm{mM}$ or $30 \mathrm{mM} \mathrm{MgCl}$ has been recently reported and has showed that high $\mathrm{Mg}^{2+}$ concentration might destabilize some pre-60S factor interactions (Meyer et al. 2010). These results strongly support the hypothesis that $\mathrm{Ecm} 1$ is a part of pre-60S particles and are correlated with recent data revealing that $\mathrm{Ecm} 1$ is present in fractions corresponding to the 60S peak ( $\mathrm{Li}$ et al. 2009). However, Ecml fused to TAP or 3HA tag was probably weakly associated with the particles because the association was loosened by extensive washing in batches instead of washing on columns (Mobicol) during the TAP purification (data not shown), and association in the sucrose gradient was dependent on the $\mathrm{Mg}^{2+}$ concentration.

\section{Ecm 1 is a shuttling protein re-imported by the kap123 Karyopherin}

$\mathrm{Ecm} 1$ is weakly associated with the pre-60S particles, and the depletion of Ecm 1 combined with the deletion of genes coding for NPC components led to an accumulation of pre-60S particles into the nucleus. In addition, $\mathrm{Ecm} 1$ is mainly localized into the nucleus with a faint signal in the cytoplasm. These observations suggested that Ecm 1 could be a shuttling protein that accompanies pre-60S particles during nuclear export. To assess Ecm1 shuttling, we performed a heterokaryon assay using the kar1-1 strain (Vallen et al. 1992). We used Arx1 and Garl as positive and 
TABLE 1. Proteins associated with Ecm1-TAP

\begin{tabular}{lccc}
\hline ORF & Gene & $\begin{array}{c}\text { Exp1 } \\
\text { Peptide Nbr }\end{array}$ & $\begin{array}{c}\text { Exp2 } \\
\text { Peptide Nbr }\end{array}$ \\
\hline YMR308C & KAP121 & & 11 \\
YDR395W & KAP108 & 14 & 10 \\
YPL093W & NOG1 & 8 & 14 \\
YNR053C & NOG2 & 11 & 14 \\
YLR449W & FPR4 & & 5 \\
YAL059W & ECM1 & 12 & 19 \\
YPR108W-A & YPR108W-A & 5 & \\
\hline
\end{tabular}

Proteins associated with Ecm1-TAP were separated on a polyacrylamide-SDS gel. The proteins were identified as described in Materials and Methods.

negative controls, respectively. Strains expressing Ecm1-GFP and Arx1-GFP or Gar1-GFP fusion proteins were mated with the kar1-1 strain, which is defective in nuclear fusion after mating. We observed that, in contrast to Garl-GFP, which stayed confined to the original nucleus into heterokaryons, Ecm1-GFP, like Arx1-GFP, was not restricted to the donor nucleus but appeared in both nuclei of the heterokaryons consistent with a nucleocytoplasmic shuttling of Ecm1-GFP (Fig. 4A).

Since $\mathrm{Ecm} 1$ is a shuttling factor and is physically associated with karyopherins, which act to import proteins from the cytoplasm to the nucleus, we wondered whether Ecm1 nuclear import, back to the nucleus, was dependent on these karyopherins. Strains expressing either Ecm1-GFP or Arx1GFP fusion proteins in which KAP121 or KAP123 was under the control of the GAL1 promoter were shifted to repressive glucose conditions for $8 \mathrm{~h}$. After Kap121 depletion, Ecm1 import was not affected, whereas Arxl-GFP accumulated into the cytoplasm as previously described (Fig. 4B; Lebreton et al. 2006). In contrast, when Kap123 was depleted or deleted (data not shown), Ecm1-GFP accumulated in the cytoplasm, whereas Arx1-GFP localization was not affected (Fig. 4B). These results indicate that Ecm1 and Arx1 are imported into the nucleus via different karyopherins.

\section{Ecm1 is physically associated with nuclear pore components}

Since several lines of evidence suggested that $\mathrm{Ecm} 1$ could be a pre-60S export factor, we checked for physical interaction between Ecml and the NPC, by performing a large-scale two-hybrid screen using Ecml as bait. In addition to the pre-60S factor Fpr4, previously identified in the Ecm1-TAP purification, we selected an Nup192 N-terminal fragment from position 2 to 493 amino acids interacting with $\mathrm{Ecm} 1$ (Table 2). We confirmed the interaction between Ecml and Nup192 by a two-hybrid matrix analysis (Fig. 5). The decapping factor Edc3 was used as a negative control. Even if the physical interaction identified by two-hybrid screen between Ecm1 and Nup192 was unambiguous, it does not prove that the interaction is direct. To check for a direct interaction, we performed an in vitro GST binding assay. We failed to observe a direct in vitro interaction between Ecm1 and Nup192. However, we have no proof that the purified Nup192 has a native conformation. As previously described, Nup100 physically interacted with Arx1 (Allen et al. 2002; Bradatsch et al. 2007; Hung et al. 2008) but not with Ecm1 in a two-hybrid and in an in vitro GST interaction assay (data not shown).

Altogether, these results strengthen the idea that $\mathrm{Ecm} 1$, as Arxl, binds to the NPC but probably to different nucleoporins.

\section{Ecm1 interferes with other pre-60S export pathways}

Since Ecm 1 behaves like a factor involved in pre-60S particle export, we wondered if the three pre-60S export receptor mutants previously described could be synthetic

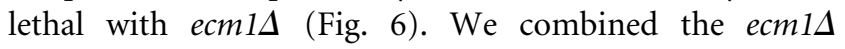

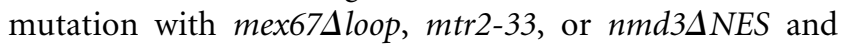
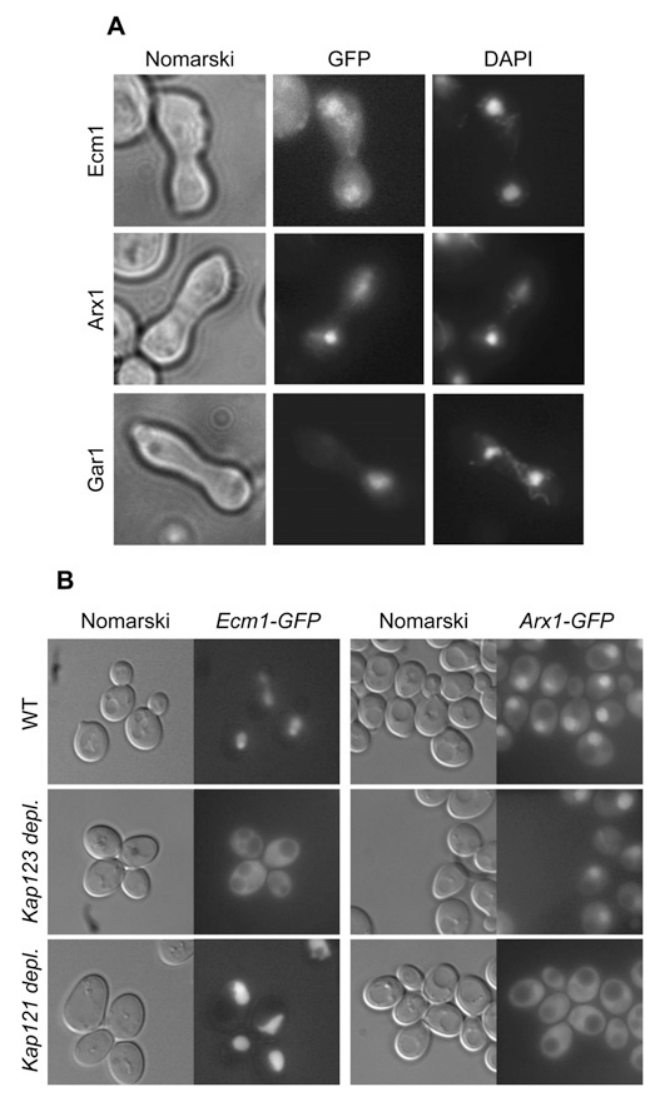

FIGURE 4. Ecm1 is a shuttling protein reimported by Kap123. (A) Ecml-GFP is present in both nuclei of heterokaryons. Cells expressing Ecm1-GFP, Arx1-GFP, or Garl-GFP fusion proteins were mated with karl-1 cells. After staining of the heterokaryons with DAPI, GFP signal and nuclei were observed by fluorescence microscopy. (B) Ecml-GFP accumulates in the cytoplasm of yeast depleted for Kap123. Strains in which KAP121 or KAP123 were expressed under the control of the GAL1 promoter and a wild-type strain producing either Ecm1-GFP or Arx1-GFP were used. After growth in galactosecontaining medium, the cultures were shifted into glucose-containing medium for $8 \mathrm{~h}$, and the GFP signal was visualized. 
TABLE 2. Proteins associated with Ecm $12 \mathrm{H}$ bait

\begin{tabular}{|c|c|c|}
\hline Gene & $\begin{array}{c}\text { Number of clones } \\
\text { (number of independent fusions) }\end{array}$ & Description \\
\hline ADE8 & $2(1)$ & Adenine biosynthesis \\
\hline FPR4 & $6(3)$ & Potential pre-60S factor \\
\hline GPA2 & $4(1)$ & Response nutriment \\
\hline NTE1 & $6(2)$ & Phosphatidylcholine synthesis \\
\hline NUP192 & $2(1)$ & Nucleoporin \\
\hline RET2 & $2(2)$ & $\begin{array}{l}\text { Transport between Golgi and ER COPI } \\
\text { complex }\end{array}$ \\
\hline SEC6 & $3(1)$ & Exocyst complex \\
\hline SPA2 & $2(1)$ & Polarisome actin cytoskeletal organization \\
\hline SSQ1 & $2(1)$ & $\begin{array}{l}\text { Mitochondrial hsp70-type molecular } \\
\text { chaperone }\end{array}$ \\
\hline STH1 & $3(3)$ & RSC chromatin remodeling complex \\
\hline TRM10 & $2(1)$ & tRNA methyltransferase \\
\hline YPLO09C & $13(2)$ & Unknown function \\
\hline
\end{tabular}

Only the candidates found at least twice are indicated. mutant, respectively (see pFL44-ECM1 into $P_{G A L 1}-A R X 1$ pom34A and pFL44$A R X 1$ into $P_{G A L 1}-E C M 1$ pom34A). ECM1 overexpression did not complement the slow growth phenotype of an arxis strain (data not shown).

$\mathrm{Ecm} 1$ and Arx1 thus share functional interactions with the NPC in a process that also involves MEX67. However, Ecml and Arxl seem to have some non-overlapping roles in pre-60S export.

\section{DISCUSSION}

\section{Ecm1 is involved in 605 particle export}

The involvement of Ecm1 in ribosome biogenesis is supported by several re$P_{G A L 1}$-ECM1 mutation with $x p o 1-1$ to test the pairwise functional interactions. When $\mathrm{Ecm} 1$ was absent and another pre-60S export pathway was defective ( $\mathrm{Nmd} 3 / \mathrm{Xpo1}$, Mex67/Mtr2), we observed an aggravating growth phenotype for all the double mutants (Fig. 6). Consistent with this observation, ecmld did not exhibit a genetic link with $m$ tr2-21, which impairs only mRNA export. These results perfectly match with unpublished data that were first obtained in Ed Hurt's laboratory (University of Heidelberg) (B Bradatsch, J Bassler, and E Hurt, pers. comm.).

To identify additional proteins functionally linked with ECM1, we performed a multicopy suppressor screen using the $P_{\text {GAL1 }}$-ECM1 arx $1 \Delta$ double-mutant strain. In addition to plasmid-containing, as expected, ECM1 or ARX1, only MEX67, was found as a multicopy suppressor of the $P_{G A L 1^{-}}$ ECM1 arx1s strain. Previous studies have shown that MEX67 overexpression could rescue an arx1 $1 \Delta$ slow-growth phenotype (Hung et al. 2008). To confirm the screen results, we transformed the three double-mutant strains with ECM1, ARX1, MEX67, or POM34overexpressing plasmids. Serial dilutions on minimal medium containing glucose without uracil (-URA) were performed. Interestingly, MEX67 overexpression was able to partially complement the growth of the three double-mutant strains (Fig. 7A).

We tested whether this growth complementation was associated with the rescue of the pre-60S particle export in this condition (after $17 \mathrm{~h}$ of growth in nonpermissive culture conditions). We observed that MEX67 overexpression better rescued the export of pre-60S in the $P_{G A L 1^{-}}$ ECM1 pom $34 \Delta$ than in the two other double mutant strains where a nuclear retention of the particles could still be detected (Fig. 7B). This is in good agreement with the observation of a better rescue of the $P_{G A L 1}$-ECM1 pom $34 \Delta$ growth defect by MEX67 overexpression.

In contrast, neither ECM1 nor ARX1 overexpression was able to complement Arx1 or Ecm1 depletion in the double sults. ECM1 expression is modulated similarly to genes coding for preribosomal factors in response to various environmental stresses (Gasch et al. 2000). ECM1 deletion has no visible effect on growth in standard conditions, but becomes essential for pre-60S particles biogenesis when the NPC is affected or in the absence of Arxl. This leads to a decrease of the amount of $60 \mathrm{~S}$ subunit, to the presence of halfmers, and to an accumulation of 27SB rRNA that reveals a weak defect on rRNA maturation, which is possibly indirect. Moreover, in addition to many large subunit ribosomal proteins, $\mathrm{Ecm} 1$ is associated with pre$60 \mathrm{~S}$ factors such as Nog1, Nog2, and Fpr4. It co-sediments with the $60 \mathrm{~S}$ peak as previously reported ( $\mathrm{Li}$ et al. 2009).

In comparison with other pre-60S factors, Ecm 1 appears weakly associated with pre-60S and thus escaped to previous observation in large-scale TAP experiments. Moreover,

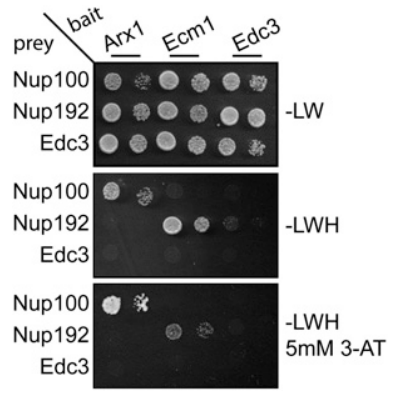

FIGURE 5. Ecm1 physically interacts with Nup192, whereas Arx1 interacts with Nup100. CG1945 yeast cells transformed with the bait plasmids (Arx1, Ecm1, or Edc3) were mated with Y187 yeast cells transformed with the prey plasmids (Edc3, Nup192 fragment [2-493], and Nup100 fragment [1-588]). The mRNA decapping factor Edc3 was used as negative control. After selection of the diploids on minimal medium plates without leucine and tryptophan (-LW), the interactions were identified on minimal medium without leucine, tryptophan, and histidine $(-\mathrm{LWH})$ supplemented or not with 3-AminoTriazol (5 mM 3-AT). Two different cells dilutions were spotted on selected plates. 
A

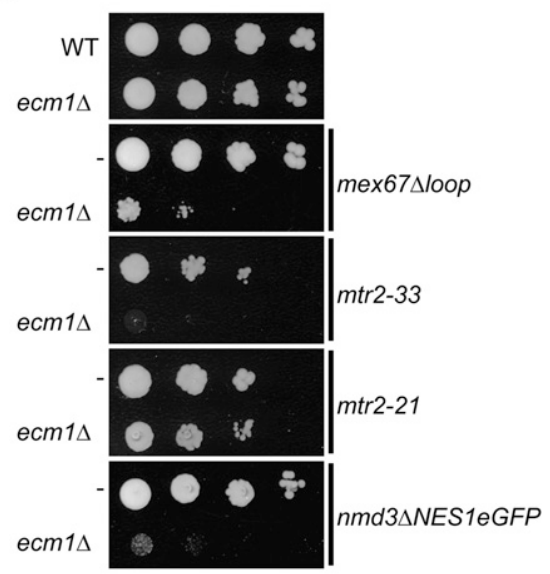

B

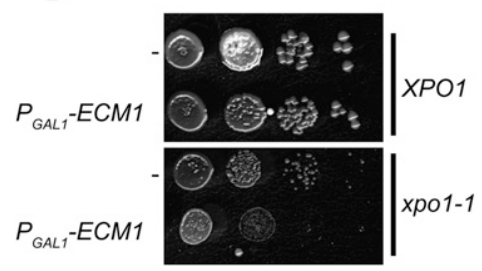

FIGURE 6. ECM1 is functionally linked to the pre-60S export receptors. (A) ecm $1 \Delta$ is synthetic lethal or synthetic slow growth with the pre-60S export process. Deletion of ECM1 is combined with several mutant alleles of export receptor genes (mex67 1 loop, $m$ tr2-33, $m t r 2-21$, or $n m d 3 \Delta N E S e G F P$ ). Serial dilutions of mutants were spotted on 5-FOA-containing plates to shuffle the plasmids carrying the wild-type genes. Strains carrying the wild-type ECM1 allele are indicated by (-). Plates were incubated for $3 \mathrm{~d}$ at $30^{\circ} \mathrm{C}$. (B) $P_{G A L 1^{-}}$ ECM1 was combined with xpo1-1 mutant, and serial dilutions of the strains were spotted on rich medium plates containing glucose. Strains carrying the wild-type ECM1 allele are indicated by (-). The growth phenotype was observed after incubation of the plates for $3 \mathrm{~d}$ at $30^{\circ} \mathrm{C}$.

Ecm 1 interacts physically with karyopherins such as Kap121, Kap108, and Kap123, suggesting that Ecm1 could play a particular role in the nuclear import of preribosomal or ribosomal factors. We explored a putative role of Ecm 1 in nuclear pre-60S factors or ribosomal protein import by looking at the cellular localization of GFP fusions for pre$60 \mathrm{~S}$ factors such as Nog1 (data not shown) and Nog2 (Supplemental Fig. 2) or by using the Rpl25NLS-GFP reporter (Supplemental Fig. 3; Timney et al. 2006). Their localizations remained unchanged in the absence of Ecm 1 . It is thus likely that Ecm 1 is a pre-60S export factor rather than involved in pre-60S factor import, in agreement with its interaction with pre-60S particles. In support of this hypothesis, we observed that when Ecml was depleted and the NPC was deficient, Rpl25-eGFP accumulated in the nucleus, revealing a block in the export of pre-60S particles from the nucleus. In addition, ECM1 is functionally linked to several pre-60S export receptors. We found that ecm $1 \Delta$ is synthetic lethal with $\operatorname{arx} 1 \Delta, m t r 2-33$, mex67delta loop, and with nmd3deltaNES, consistent with independent and similar results observed in Ed Hurt's laboratory (University of Heidelberg). They showed that ecm1 $\Delta$ is synthetic lethal with the mtr2-33, which impaired pre-60S export, but not with the mtr2-21 allele, which impaired mRNA export (Bassler et al. 2001), with mex67delta loop and with nmd3deltaNES, but not with $\mathrm{N}$-terminal mutants like $n m d 3-2, n m d 3-150$, and nmd3-169 (B Bradatsch, J Bassler, and E Hurt, pers. comm.). Similar functional links observed between arx $1 \Delta$ and the same mutant alleles of export receptor genes (Bradatsch et al. 2007; Hung et al. 2008) provide additional arguments in favor of a role of ECM1 in pre-60S export.

In summary, Ecm 1 is not essential but is involved in pre$60 \mathrm{~S}$ export, becoming essential to this process when the nuclear pores are affected or when export receptors like Arx1, Mex67/Mtr2, or Crm1 are absent or mutated. This function needs an efficient re-import of shuttling Ecml into the nucleus, probably mediated by Kap123.
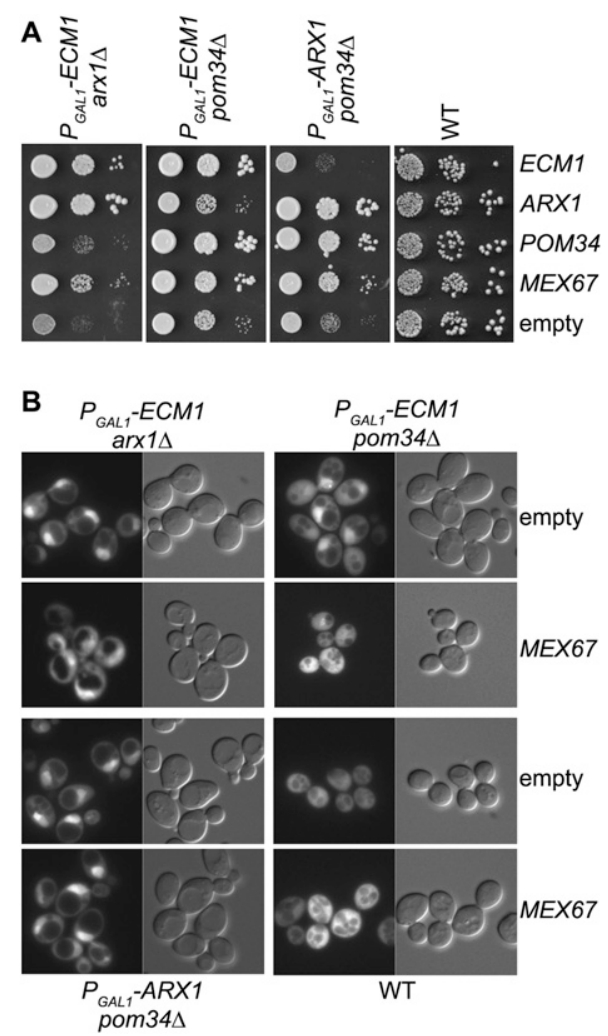

FIGURE 7. MEX67 overexpression partially complements the doublemutant strain growth defects. (A) MEX67 but not ECM1 or ARX1 overexpression partially complements the double-mutant strain growth defects. The double mutants $\left(P_{G A L 1}-E C M 1\right.$ arxis, $P_{G A L 1^{-}}$

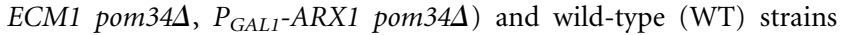
were transformed with either empty pFL44L vector $(-)$ or pFL44L containing either ECM1, ARX1, POM34, or MEX67. Transformants were spotted in steps of 10 -fold dilution on minimal medium without uracil and incubated for $2 \mathrm{~d}$ at $30^{\circ} \mathrm{C}$. (B) MEX67 overexpression partially restores pre-60S subunit export in the $P_{G A L 1}$-ECM1 pom $34 \Delta$ mutant strain. Subcellular localization of Rpl25-eGFP was observed by fluorescence microscopy in the mutant and WT strains transformed with high-copy vectors expressing ARX1, ECM1, POM34, or MEX67. 


\section{Coordinated transport and redundancy}

In eukaryotic cells, nuclear pores control and synchronize nuclear and cytoplasmic events. The ribosome main function is cytoplasmic, but a large part of its maturation takes place within the nucleus. According to the cell status, the nuclear pores must not only control the import of proteins required for rRNA maturation, but also the export of ribosome precursors at well-defined steps. In addition, ribosome biogenesis is highly regulated and must quickly be repressed or induced in response to environmental changes. Consequently, this pathway needs the cooperation of several distinct pathways such as mRNA export and ribosomal subunit export. Three export receptors were previously described. Two of them, Crm1 and Mex67Mtr2, which are also essential general export receptors, could be involved in this process. Recent studies showed that Mex67-Mtr2, initially described to be essential for mRNA export (Segref et al. 1997; Santos-Rosa et al. 1998), is also involved in preribosomal particle export (Yao et al. 2007). These results might indicate a cross-talk between mRNA export and ribosome export pathways. We performed an mRNA export assay using Cy3-labeled oligo(dT) to follow the localization of poly $(\mathrm{A})^{+}$mRNA. We observed no mRNA accumulation in the nucleus in the absence of $\mathrm{Ecm} 1$, consistent with the fact that $\mathrm{Ecm} 1$ is required for pre$60 S$ export rather than mRNA export (Supplemental Fig. 4). An overexpression assay showed that the mex674loop allele, deficient for large ribosomal subunit export, was not able to complement the growth defect of the double mutant $P_{G A L 1^{-}}$ ECM1 arx1A, in contrast to the overexpression of MEX67 (Supplemental Fig. 5). This result also supports a role of Ecm1 for pre-60S export rather than mRNA export.

It is also important to maintain the stoichiometry between mature small and large subunits. Even if the pre- $40 \mathrm{~S}$ and pre-60S particle exports are independent from one another, the production of both subunits must be equivalent (Johnson et al. 2002; Tschochner and Hurt 2003; Zemp and Kutay 2007). The involvement of a common export receptor Crm1 for both pre-ribosomal particles (Moy and Silver 1999; Ho et al. 2000; Gadal et al. 2001; Moy and Silver 2002) may ensure the coordination of an equilibrated export of both subunits. Data from large-scale synthetic lethal screens revealed an intriguing link between $S Q S 1$, coding for a component of the pre-40S ribosomal particles, and ECM1 (Decourty et al. 2008). This genetic interaction might reveal interplay between both pre-40S and pre-60S biogenesis.

Due to their large size, it is expected that pre-ribosomal subunit export might require several export factors. Arx1, Mex67, and Nmd3 have been found associated with $\mathrm{Ecm} 1$ containing pre-60S particles (data not shown); however, it has not yet been formally proven that all four factors are present simultaneously in the same particle. In addition, even if these four export factors are functionally linked, they are not fully redundant. We and others have shown that the overexpession of MEX67 is able to partially restore the phenotype of the three other export factor mutants (Bradatsch et al. 2007; Hung et al. 2008), whereas the overexpression of ECM1 is not able to complement the absence of Arx1. These results suggest that the large ribosomal export requires both essential general export factors like Crm1 and Mex67/Mtr2 and specific pre-60S factors like Arx1 and Ecm1.

\section{MATERIAL AND METHODS}

\section{Strains and plasmids}

Yeast strains used in this study are listed in Supplemental Table 1. Conditional mutant strains and fusion genes were generated by homologous recombination using PCR products synthesized from plasmid templates (Longtine et al. 1998).

Plasmids for bacterial protein production and for two-hybrid assays were constructed using the Gateway system (Invitrogen). pFL44L-containing plasmids ECM1, POM34, ARX1, and MEX67 were constructed using a TOPO cloning vector as intermediate. Each gene was cloned between SacI and NotI restriction sites. The boundaries upstream of the ATG and downstream from the Stop codon for each gene are the following: ECM1 (-178; +207); POM34 (-192; +112); ARX1 (-108; +162); and MEX67 (-204; +179 ). Plasmid pFL44L containing mex674loop was obtained by gap repair with a PCR fragment from pRS314-mex674loop (a gift from Ed Hurt's laboratory [University of Heidelberg]). The plasmids used in this study are listed in Supplemental Table 2.

\section{Polysome gradient}

The strains were grown to $A_{600 \mathrm{~nm}}$ around 0.6 in rich medium containing either galactose or glucose. To observe the polysome profile (Fig. 2B), the lysates were prepared in $20 \mathrm{mM}$ Tris- $\mathrm{HCl}$ (pH 7.4), $30 \mathrm{mM} \mathrm{MgCl}, 100 \mathrm{mM} \mathrm{NaCl}$, and $50 \mu \mathrm{g} / \mathrm{mL}$ cycloheximide using glass bead vortexing. They were fractioned by ultracentrifugation on a $10 \%-50 \%$ sucrose gradient in a buffer containing $20 \mathrm{mM}$ Tris- $\mathrm{HCl}$ ( $\mathrm{pH} 7.4$ ), $30 \mathrm{mM} \mathrm{MgCl}_{2}, 50 \mathrm{mM}$ $\mathrm{NH}_{4} \mathrm{Cl}$, and $1 \mathrm{mM}$ DTT for $2 \mathrm{~h} 45 \mathrm{~min}$ at $39,000 \mathrm{rpm}$ at $4^{\circ} \mathrm{C}$ (SW41 rotor). To look at the association of Ecm1 with the pre-60S particles (Fig. 3C), the lysates were prepared in $20 \mathrm{mM}$ Tris ( $\mathrm{pH}$ 7.4), $50 \mathrm{mM} \mathrm{KCl}, 5 \mathrm{mM} \mathrm{MgCl}_{2}$, and $50 \mu \mathrm{g} / \mathrm{mL}$ cycloheximide and fractionated by ultracentrifugation on a $10 \%-50 \%$ sucrose gradi-

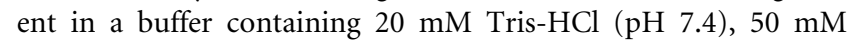
$\mathrm{NH}_{4} \mathrm{Cl}, 5 \mathrm{mM} \mathrm{MgCl}_{2}$, and $1 \mathrm{mM}$ DTT as described by Meyer et al. (2010).

\section{Affinity purification}

The tandem affinity purifications were performed starting with $4 \mathrm{~L}$ of yeast culture at an $A_{600} \mathrm{~nm}$ around 1 as described by Rigaut et al. (1999). Washing and elution steps were performed in Mobicols (Mo Bi Tec). Eluted proteins were precipitated with $10 \%$ TCA and separated on a $4 \%-12 \%$ polyacrylamide gradientSDS gel. Proteins were stained with colloidal Coomassie Blue. Equally spaced, 2-mm bands were cut, and proteins were subjected to in gel tryptic digestion. The peptides were analyzed 
with a 4800 MALDI TOF/TOF analyzer (Applied Biosystems). Protein identifications were performed by searching against a database containing the entire $S$. cerevisiae proteome with MS/MS spectra (0.3 Da precision) using the MASCOT software (Matrix Science).

\section{Western blot analysis}

The proteins were separated on $4 \%-12 \%$ polyacrylamide gradient-SDS gels and transferred to a nitrocellulose membrane (BioRad). 3HA-tagged protein was revealed with a 1:500 dilution of anti-HA-peroxidase (Roche). Pre-ribosomal and ribosomal proteins were detected by indirect immunoblotting, using specific polyclonal rabbit antibodies at a 1:5000 dilution. Secondary antibodies (Goat Anti-Rabbit-HRP Conjugate from Bio-Rad) were used at a 1:10,000 dilution. Peroxidase activity of the secondary antibodies was visualized with the Millipore chemiluminescence HRP substrate system.

\section{High-copy suppressor screens}

The $P_{G A L 1}-E C M 1$ arx $1 \Delta$ strain was transformed with a yeast genomic library cloned into a pFL44L URA3 plasmid. The transformed cells were incubated for $3 \mathrm{~h}$ in liquid YPGal medium and then spread on solid minimum media containing glucose and lacking uracil. After $2 \mathrm{~d}$ at $30^{\circ} \mathrm{C}$, the colonies growing faster than the background were selected. The clones rescued by plasmids coding for either ECM1 or ARX1 were identified by PCR on yeast colonies using specific oligonucleotides and eliminated. The other suppressor plasmids were recovered and verified by re-transformation of the three double-mutant strains. Their growth phenotypes were compared to the same strain transformed with the empty vector. The plasmid DNAs were extracted and DNA inserts were sequenced using M13 forward and reverse universal primers.

The growth rescue effects were compared by spotting serial dilution of transformed yeast cell cultures on minimal medium containing glucose without uracil.

\section{Yeast two-hybrid experiments}

The two-hybrid screen was performed according to the method of Fromont-Racine et al. (1997). The CG1945 transformed with the pAS2 $\Delta \Delta$-ECM1 bait plasmid was mated with Y187 transformed with the FRYL genomic DNA library cloned into pActIIst. Among the 48 million diploids, $94 \mathrm{His}^{+} \mathrm{LacZ}^{+}$colonies were selected. Genomic inserts cloned into the prey plasmid were identified by sequencing. Candidates found only once or corresponding to antisense transcripts were discarded.

The two-hybrid matrix was performed with Y187 and CG1945 strains transformed with the prey and bait plasmids, respectively. $A R X 1, E C M 1$, and EDC3 were cloned as entire ORF sequences into the pAS2 $\Delta \Delta$ bait plasmid. The NUP192 fragment (amino acids 2-493) was selected as a prey partner in the two-hybrid screen, and EDC3 was cloned as the entire ORF into the prey plasmid. The two latter ORFs were cloned into a pActIIst prey plasmid. Both transformed strains were mated, and diploids were selected on minimal medium without leucine and tryptophan. The interactions were identified on minimal medium without leucine, tryptophan, and histidine supplemented or not with 3-AminoTriazol at various concentrations from $1 \mathrm{mM}$ to $25 \mathrm{mM}$.

\section{Fluorescence microscopy}

Yeast cells were grown in minimal media overnight at $30^{\circ} \mathrm{C}$ to an $A_{600 \mathrm{~nm}}$ of 0.4 . Double-mutant strains were incubated in minimal medium containing galactose and then shifted to glucose minimal medium for different times depending on the strains. Fluorescence was visualized using an epifluorescence microscope (model DMRA; Leica).

\section{Heterokaryon assay}

Cells were incubated in liquid YPD medium at $30^{\circ} \mathrm{C}$ until an $A_{600}$ $\mathrm{nm}$ around 1. For mating, $1 \mathrm{~mL}$ of the karl-1 strain (Vallen et al. 1992) was mixed with $1 \mathrm{~mL}$ of Ecm1-GFP, Arx1-GFP, or Gar1GFP expressing cells. The mixture was concentrated on a YPD plate to allow conjugation. After $1 \mathrm{~h}$ on a YPD plate followed by $2 \mathrm{~h}$ on YPD containing $50 \mu \mathrm{g}$ of cycloheximide per milliliter, cells were collected and fixed in $4 \%(\mathrm{v} / \mathrm{v})$ formaldehyde and analyzed by microscopy (model DMRA; Leica). Similar results were obtained with or without treatment with cycloheximide.

\section{SUPPLEMENTAL MATERIAL}

Supplemental material can be found at http://www.rnajournal.org.

\section{ACKNOWLEDGMENTS}

We thank Cécile Prud'homme for initiating the work on Ecm1. We are very grateful to Miguel Remacha for the Mrt4 antibody; to John Woolford for the Nop7 antibody; to François Lacroute for the Rpl1 antibody and for the genomic library in pFL44L used for the high copy number suppressor screen; to Michael Rout for the Rpl25NLS-GFP reporter to test the import of ribosomal proteins; to Michael Rexach for the Nup100 two-hybrid bait plasmid; and to Mark D. Rose for the kar1-1 mutant strain. We thank Betina Bradatsch, Jochen Bassler, and Ed Hurt for communicating unpublished results and for the export receptor mutant strains and plasmids. We thank Georges Lutfalla and Gwenael Breard for critical reading of the manuscript. This work was supported by grant ANR-06-BLAN-0037 from the Ministère de l'Enseignement Supérieur et de la Recherche and l'Association pour la Recherche sur le Cancer (33.49). Y.Y. received a fellowship from Le Ministère de l'Enseignement Supérieur et de la Recherche, and E.D. received fellowships from La Ligue Nationale contre le Cancer and from la Fondation pour la Recherche Médicale.

Received November 16, 2009; accepted February 10, 2010.

\section{REFERENCES}

Alber F, Dokudovskaya S, Veenhoff LM, Zhang W, Kipper J, Devos D, Suprapto A, Karni-Schmidt O, Williams R, Chait BT, et al. 2007a. Determining the architectures of macromolecular assemblies. Nature 450: 683-694.

Alber F, Dokudovskaya S, Veenhoff LM, Zhang W, Kipper J, Devos D, Suprapto A, Karni-Schmidt O, Williams R, Chait BT, et al. 2007b. The molecular architecture of the nuclear pore complex. Nature 450: 695-701.

Allen NP, Patel SS, Huang L, Chalkley RJ, Burlingame A, Lutzmann M, Hurt EC, Rexach M. 2002. Deciphering networks of protein interactions at the nuclear pore complex. Mol Cell Proteomics 1: 930-946. 
Bassler J, Grandi P, Gadal O, Lessmann T, Petfalski E, Tollervey D, Lechner J, Hurt E. 2001. Identification of a $60 \mathrm{~S}$ pre-ribosomal particle that is closely linked to nuclear export. Mol Cell 8: 517-529.

Bradatsch B, Katahira J, Kowalinski E, Bange G, Yao W, Sekimoto T, Baumgartel V, Boese G, Bassler J, Wild K, et al. 2007. Arx1 functions as an unorthodox nuclear export receptor for the $60 \mathrm{~S}$ pre-ribosomal subunit. Mol Cell 27: 767-779.

Decourty L, Saveanu C, Zemam K, Hantraye F, Frachon E, Rousselle JC, Fromont-Racine M, Jacquier A. 2008. Linking functionally related genes by sensitive and quantitative characterization of genetic interaction profiles. Proc Natl Acad Sci 105: 5821-5826.

Demoinet E, Jacquier A, Lutfalla G, Fromont-Racine M. 2007. The Hsp40 chaperone Jjj1 is required for the nucleo-cytoplasmic recycling of preribosomal factors in Saccharomyces cerevisiae. RNA 13: $1570-1581$.

Dragon F, Gallagher JE, Compagnone-Post PA, Mitchell BM, Porwancher KA, Wehner KA, Wormsley S, Settlage RE, Shabanowitz J, Osheim Y, et al. 2002. A large nucleolar U3 ribonucleoprotein required for $18 \mathrm{~S}$ ribosomal RNA biogenesis. Nature 417: 967-970.

Fahrenkrog B, Aebi U. 2003. The nuclear pore complex: Nucleocytoplasmic transport and beyond. Nat Rev Mol Cell Biol 4: 757-766.

Fromont-Racine M, Rain JC, Legrain P. 1997. Toward a functional analysis of the yeast genome through exhaustive two-hybrid screens. Nat Genet 16: 277-282.

Fromont-Racine M, Senger B, Saveanu C, Fasiolo F. 2003. Ribosome assembly in eukaryotes. Gene 313: 17-42.

Gadal O, Strau BD, Kessl J, Trumpower B, Tollervey D, Hurt E. 2001. Nuclear export of $60 \mathrm{~S}$ ribosomal subunits depends on Xpolp and requires a nuclear export sequence-containing factor, Nmd3p, that associates with the large subunit protein Rpl10p. Mol Cell Biol 21: 3405-3415.

Gasch AP, Spellman PT, Kao CM, Carmel-Harel O, Eisen MB, Storz G, Botstein D, Brown PO. 2000. Genomic expression programs in the response of yeast cells to environmental changes. Mol Biol Cell 11: 4241-4257.

Gavin AC, Aloy P, Grandi P, Krause R, Boesche M, Marzioch M, Rau C, Jensen LJ, Bastuck S, Dumpelfeld B, et al. 2006. Proteome survey reveals modularity of the yeast cell machinery. Nature 440: 631-636.

Grandi P, Rybin V, Bassler J, Petfalski E, Strauss D, Marzioch M, Schafer T, Kuster B, Tschochner H, Tollervey D, et al. 2002. 90S pre-ribosomes include the $35 \mathrm{~S}$ pre-rRNA, the U3 snoRNP, and $40 \mathrm{~S}$ subunit processing factors but predominantly lack $60 \mathrm{~S}$ synthesis factors. Mol Cell 10: 105-115.

Henras AK, Soudet J, Gerus M, Lebaron S, Caizergues-Ferrer M, Mougin A, Henry Y. 2008. The post-transcriptional steps of eukaryotic ribosome biogenesis. Cell Mol Life Sci 65: 2334-2359.

Ho JH, Kallstrom G, Johnson AW. 2000. Nmd3p is a Crmlpdependent adapter protein for nuclear export of the large ribosomal subunit. J Cell Biol 151: 1057-1066.

Hung NJ, Lo KY, Patel SS, Helmke K, Johnson AW. 2008. Arx1 is a nuclear export receptor for the $60 \mathrm{~S}$ ribosomal subunit in yeast. Mol Biol Cell 19: 735-744.

Johnson AW, Lund E, Dahlberg J. 2002. Nuclear export of ribosomal subunits. Trends Biochem Sci 27: 580-585.

Kessler MM, Zeng Q, Hogan S, Cook R, Morales AJ, Cottarel G. 2003. Systematic discovery of new genes in the Saccharomyces cerevisiae genome. Genome Res 13: 264-271.

Lebreton A, Saveanu C, Decourty L, Rain JC, Jacquier A, FromontRacine M. 2006. A functional network involved in the recycling of nucleocytoplasmic pre-60S factors. J Cell Biol 173: 349-360.
Li Z, Lee I, Moradi E, Hung NJ, Johnson AW, Marcotte EM. 2009. Rational extension of the ribosome biogenesis pathway using network-guided genetics. PLoS Biol 7: e1000213. doi: 10.1371/ journal.pbio.1000213.

Longtine MS, McKenzie A III, Demarini DJ, Shah NG, Wach A, Brachat A, Philippsen P, Pringle JR. 1998. Additional modules for versatile and economical PCR-based gene deletion and modification in Saccharomyces cerevisiae. Yeast 14: 953-961.

Meyer AE, Hoover LA, Craig EA. 2010. The cytosolic J-protein, Jjj1, and Reil function in the removal of the pre-60 S subunit factor Arx1. J Biol Chem 285: 961-968.

Moy TI, Silver PA. 1999. Nuclear export of the small ribosomal subunit requires the ran-GTPase cycle and certain nucleoporins. Genes \& Dev 13: 2118-2133.

Moy TI, Silver PA. 2002. Requirements for the nuclear export of the small ribosomal subunit. J Cell Sci 115: 2985-2995.

Rigaut G, Shevchenko A, Rutz B, Wilm M, Mann M, Seraphin B. 1999. A generic protein purification method for protein complex characterization and proteome exploration. Nat Biotechnol 17: 1030-1032.

Rout MP, Aitchison JD, Suprapto A, Hjertaas K, Zhao Y, Chait BT. 2000. The yeast nuclear pore complex: Composition, architecture, and transport mechanism. J Cell Biol 148: 635-651.

Santos-Rosa H, Moreno H, Simos G, Segref A, Fahrenkrog B, Pante N, Hurt E. 1998. Nuclear mRNA export requires complex formation between Mex67p and Mtr2p at the nuclear pores. Mol Cell Biol 18: 6826-6838.

Saveanu C, Namane A, Gleizes PE, Lebreton A, Rousselle JC, NoaillacDepeyre J, Gas N, Jacquier A, Fromont-Racine M. 2003. Sequential protein association with nascent $60 \mathrm{~S}$ ribosomal particles. Mol Cell Biol 23: 4449-4460.

Segref A, Sharma K, Doye V, Hellwig A, Huber J, Luhrmann R, Hurt E. 1997. Mex67p, a novel factor for nuclear mRNA export, binds to both poly $(\mathrm{A})^{+}$RNA and nuclear pores. $E M B O J$ 16: 3256 3271.

Sydorskyy Y, Dilworth DJ, Yi EC, Goodlett DR, Wozniak RW, Aitchison JD. 2003. Intersection of the Kap123p-mediated nuclear import and ribosome export pathways. Mol Cell Biol 23: 20422054.

Timney BL, Tetenbaum-Novatt J, Agate DS, Williams R, Zhang W, Chait BT, Rout MP. 2006. Simple kinetic relationships and nonspecific competition govern nuclear import rates in vivo. J Cell Biol 175: 579-593.

Tran EJ, Wente SR. 2006. Dynamic nuclear pore complexes: Life on the edge. Cell 125: 1041-1053.

Tschochner H, Hurt E. 2003. Pre-ribosomes on the road from the nucleolus to the cytoplasm. Trends Cell Biol 13: 255-263.

Vallen EA, Hiller MA, Scherson TY, Rose MD. 1992. Separate domains of KAR1 mediate distinct functions in mitosis and nuclear fusion. J Cell Biol 117: 1277-1287.

Venema J, Tollervey D. 1999. Ribosome synthesis in Saccharomyces cerevisiae. Annu Rev Genet 33: 261-311.

Warner JR. 1999. The economics of ribosome biosynthesis in yeast. Trends Biochem Sci 24: 437-440.

Yao W, Roser D, Kohler A, Bradatsch B, Bassler J, Hurt E. 2007. Nuclear export of ribosomal $60 \mathrm{~S}$ subunits by the general mRNA export receptor Mex67-Mtr2. Mol Cell 26: 51-62.

Yao W, Lutzmann M, Hurt E. 2008. A versatile interaction platform on the Mex67-Mtr2 receptor creates an overlap between mRNA and ribosome export. EMBO J 27: 6-16.

Zemp I, Kutay U. 2007. Nuclear export and cytoplasmic maturation of ribosomal subunits. FEBS Lett 581: 2783-2793. 

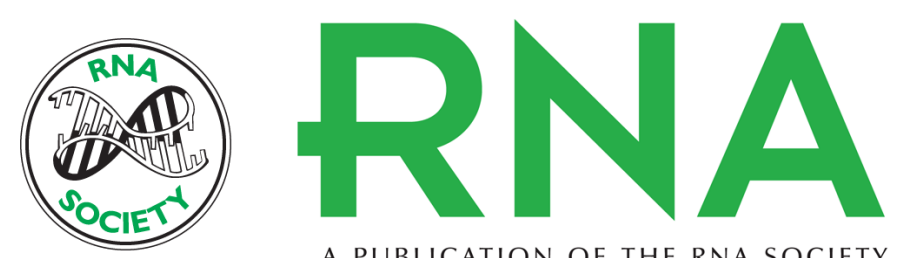

A PUBLICATION OF THE RNA SOCIETY

\section{Ecm1 is a new pre-ribosomal factor involved in pre-60S particle export}

Yanhua Yao, Emilie Demoinet, Cosmin Saveanu, et al.

RNA 2010 16: 1007-1017 originally published online March 26, 2010

Access the most recent version at doi:10.1261/rna.2012310

Supplemental

Material

References

\section{License}

Email Alerting

Service
http://rnajournal.cshlp.org/content/suppl/2010/03/23/rna.2012310.DC1

This article cites 40 articles, 19 of which can be accessed free at: http://rnajournal.cshlp.org/content/16/5/1007.full.html\#ref-list-1 